\title{
PARAGONIMIASIS IN MANIPUR
}

(Singh SK, Mutum S, Razaque MA, Singh YI, Singh EY, Parngonimiasis in Manipur. Ind J Med Res [A] 1993; 97: 247-252)

\section{Summary}

Objective: To determine the prevalence of paragonimiasis in Manipur using intradermal test and some of the epidemiological factors attributable to it.

Study Design: Random cross sectional study.

Patients: 3,467 individuals of both sexes aged 5 years and above were tested.

Intervention; Intradermal test with a saline extract of $P$. westarmanii in all. Skin positive reactors (234) and an equal number of negative reactors examined for symptoms and signs of disease. Seventy five dermal reactors and an equal number of non reactors were available for investigations of sputum and stool. Those whose sputa showed parasitic eggs were subjected to X-ray chest.

Main outcome measures: Difference of more than $5 \mathrm{~mm}$ between the antigen and control wheal diameters was taken as positive skin test.

Main results: The overall prevalence rate observed was $6.7 \%$, proportion of positive reactors was $8.0 \%$ among crab eaters (234/2834). 533 non crub eaters were non reactors. The differences in the prevalence among males $(9.6 \%)$ and females $(4.6 \%)$ was significant $(P<0.01)$. Higher prevalence rates were found in age groups $15-45$ years. Symptoms like cough, chest pain, and haemoptysis were significantly more frequent amangst reactors. Pragonimus eggs were seen in the sputum of four of 75 skin reactors, these four had a raised ESR of $20-59 \mathrm{~mm}$ fall in the first hour, eosinophilia of $500-1500 / \mathrm{cmm}$ and patchy opacities/cystic lesions in chest X-rays.

Conclusions: Intra dermal test is a good screening test for mass surveys of paragonimiasis. Paragonimiasis is widely distributed in Manipur. Ingestion of crabs has a significant correlation with ser opositivily.

\section{Comments:}

Paragonimiasis (ondemic haemoptysis) is a chronic infection of the lung caused by tremalodes of the genus Paragonimus. The infection is acquired by ingestion of cysts or metacercarie through uncooked crabs or cray fish, the intermediate hosts. Clinically the disease is characterised by cough and haemoptysis. The eggs are passed in sputum or faeces if swallowed.

The skin test in this study was of proven sensitivity. Cross reactions may occur with other distomes [1]. Use of a refined fraclionated antigen may exclude such cross renclions. The skin test positivity does not indicate current infection. It may remain positive for years even after complete recovery. Spontaneous cure is known in light infections with death of worms in 5-6 years [2]. These factors might have been responsible for poor recovery rate $(5.3 \%)$ of eggs.

The study has employed direct wet mounts and concentration techniques to look for eggs in sputum and stool. The best way to look for eggs in spulum is by concentrating it with $3 \% \mathrm{KOH}$ for 10 minutes and centrifugation for 5 minutes at 200 rpm. Formalin - ether concentration technique can be applied to stool. Ziehl-Neclsen stain does not demonstrate eggs in sputum or stool and may destroy the eggs that are present [3]. It should be avoided if paragonimiasis is suspected.

The drug of choice for paragonimiasis is praziquantel [2] and four egg passers werc treated with it. No follow up skin tesls have been done in them.

The study has a relevance for Armed Forces in that troops stationed in and around Manipur may acquire local eating habits and contact paragonimiasis. Clinically and radiologically paragonimiasis may mimic tuberculosis. Therefore patients who present with cough, haemoptysis and give history of eating crabs/cray fish should be investigated for pulmonary paragoniminsis before initiating anti tubercular treatment.

\section{REFERENCES}

1. Sawada T, Takci K, Kale S, Hatsuyama S. Studies on the immunodiagnosis of paragonimiasis using intradermal test with fractionated antigen. I Inf $D$ is 1968; $118: 235$.

2. Brown WH, Neva FA. Basic Clinical Parasitology. 5th Ed., Norwalk : Applcton-Cenlury-Crafts 1983; 230-1.

3. Plorde JJ. Other Trematodes and Flukes. In Harrison's Principles of Internal Medicine. Braunwald E, Isselbacher JK, Petersdorf RG, Wilson JD, Martin JB, Fauci AS eds, 11th Ed.. New-york; McGrow - Hill Book Company 1987; 822-4.

Contributed by

Dr S Joshi, Lt Col MKK Rao

Dept of Microbiology, AFMC, Pune 411040. 